\title{
Accuracy of Segmented Versus Full Arch Three Dimensionally Printed Transfer Tray for Orthodontic Indirect Bonding
}

\section{Mariam Alaa El-Sebaay ${ }^{1 *}$, Khaled Hazem Attia ${ }^{2}$, Mai Hamdi Aboul Fotouh $^{3}$ and Amr Ragab El Bialy ${ }^{4}$}

${ }^{1}$ Orthodontics Master Candidate, Department of Orthodontics, Faculty of Dentistry, Cairo University, Giza, Egypt

${ }^{2}$ Professor of Orthodontics, Faculty of Dentistry, Cairo University, Giza, Egypt

${ }^{3}$ Lecturer of Orthodontics, Faculty of Dentistry, Cairo University, Giza, Egypt

${ }^{4}$ Associate Professor of Orthodontics, Faculty of Dentistry, Cairo University, Giza,

Egypt

*Corresponding Author: Mariam Alaa El-Sebaay, Orthodontics Master Candidate, Department of Orthodontics, Faculty of Dentistry, Cairo University, Giza, Egypt.
Received: January 04, 2021

Published: April 15, 2021

(C) All rights are reserved by Mariam Alaa

El-Sebaay., et al.

\section{Abstract}

Objective: This study is done to investigate the accuracy of two novel digitally printed transfer trays (full arch and segmented) thus the problems of conventional indirect bonding technique is overcome. This accuracy is measured in terms of accuracy of transferring brackets.

Methods: Patients with mild to moderate crowding, requiring orthodontic treatment with full set of permanent teeth including second molars, will be selected for this study (7 in each group). 98 brackets for Full Arch Tray and 98 for Segment Arch Tray will be used with standardization of bracket type and bonding material both groups. Indirect bonding technique of Silverman (1972) will be used to treat patients with fixed orthodontic appliances. The modification that will be done is digital bracket placement using bracket placement module of 3 Shape Ortho planner Software (3Shape Company- Copenhagen, Denmark) and fabrication of segmented digital bracket transfer tray using bracket transfer module of same software instead of manual bracket placement directly on study model and vacuum transfer tray. For control group patients, all patients of this group will follow same steps of indirect bonding procedure as treatment group patients but the full arch tray will be replaced by segmented one. Position of brackets on pre and post-operative scans will be compared. To measure the accuracy of bracket transfer, 3 Shape Ortho planner software (Bracket Placement Module) will be used.

Results: Attachment deviations linearly were within the clinically acceptable range of deviation (+/- $0.5 \mathrm{~mm})$ in all three planes for both techniques.

Conclusion: Regarding linear directional deviation in the mesio-distal plane, occluso-gingival and bucco-lingual, no differences were shown in both techniques.

Keywords: Orthodontic Indirect Bonding; Full Arch Tray; Segment Arch Tray; Transfer Tray 


\section{Background}

The transfer tray affects greatly the accuracy of indirect bonding technique. Since 1999, different materials of transfer tray were introduced including: hybrid systems made of resin and silicone, either for full arch or segmented trays. Segmentation of the indirect bonding tray was a suggestion to reduce bond failure, and segmented tray was found to be more efficient in tray placement and controlling isolation when compared to full arch tray, and hence reduces bond failure [1]. Segmented tray was splitted either into two segments only (one for each quadrant) or three segments (one anterior and two posterior segments) for each arch.

Accuracy of bracket positioning using indirect bonding technique may be attributed to thickness of bonding material between teeth and brackets, any contamination that may occur during transfer, or any error that occurred during transfer tray fabrication. However, high accuracy of bracket positioning during transfer, was found with segmented trays, reaching $98 \%$ regarding buccolingual and mesiodistal dimension [3].

Grünheid., et al. (2015) [2], studied the transfer accuracy of vinyl polysiloxane (VPS) trays for the indirect orthodontic attachments bonding, in a clinical study, a total of 136 brackets were evaluated. The brackets were bonded on the casts with a light-cure composite adhesive and cone beam computed tomography (CBCT) was used for scanning. The tray was then fabricated with vinyl polysiloxane (VPS). A chemical cure composite sealant was applied to the etched tooth surfaces and to the individualized bracket bases after mixing. The segmented transfer trays were then seated over the teeth, one quadrant at a time, with firm finger pressure for 2 minutes to hold it in place and left for an additional 8 minutes without finger pressure to allow complete curing of the sealant. CBCT was used to scan the patient's dentition to capture the final bracket positioning on the teeth. The two sets of scans were used to construct virtual models, then digitally superimposed by the use of customized software using Best fit Superimposition Technique, for evaluation of linear differences (mesiodistal, buccolingual, and vertical dimensions) and angular differences (torque, tip, and rotation). Linear deviations less than $0.5 \mathrm{~mm}$ and angular deviations less than 2 degrees were considered clinically acceptable. Results showed that torque showed lowest transfer accuracy (80.15\%), while mesiodistal and buccolingual bracket placement showed hi- ghest results (both 98.53\%). The study concluded that the (VPS) trays, transfers the planned bracket position from the dental cast to the patient's dentition very accurately.

El Nigoumi (2016) [3] described a new technique for testing the transfer accuracy of indirectly bonded attachments in all three dimensions. The working model with the bonded attachments was lightly sprayed with titanium dioxide to avoid metallic reflections, and digitally scanned. A $5 \mathrm{~mm}$ soft sheet was vacuformed over the attachments and cast, followed by a $1 \mathrm{~mm}$ hard sheet to fabricate the transfer tray. To evaluate the accuracy of transferring the attachment to the patient's dentition after the bonding procedure, an intraoral scan was made with a PlanScan 3D Scanner. This avoids any distortion from the common tearing of impression material caused by sharp bracket wings and hooks. An STL file was exported, and Geomagic Qualify version 12.0 software was used to superimpose and analyze the scanned images in three dimensions. The author stated that, Orthodontists can use this method to evaluate their clinical techniques, ensuring consistent reproducibility of bracket positions. Researchers can test available transfer trays to determine the most accurate techniques and materials.

Reproducible technique with standard results was the aim of several studies; however, none have reached to the most reliable technique because of the human factor that is greatly involved starting from bracket placement and ending with bracket transfer using transfer tray [4]. With the evolution of 3D imaging and printing machinery, digitization was introduced recently in orthodontic field. These new technologies offer extremely high accuracy as well as elimination of errors due to human variations. Intraoral scanner devices offer numerous advantages in orthodontics such as enabling fabrication of three dimensionally printed bracket transfer tray, digital storage of study models and advanced software for bracket placement $[5,6]$. Therefore, utilization of 3D imaging and printing techniques can help the orthodontist to reach the most precise indirect bonding technique with more accurate and precise results.

This study is done to investigate the accuracy of two novel digitally printed transfer trays (full arch and segmented) thus the problems of conventional indirect bonding technique is overcome. This accuracy is measured in terms of accuracy of transferring brackets. 


\section{Material and Methods}

Study settings:

- Source of patients: Outpatients of clinic of Orthodontic department, Faculty of Dentistry, Cairo University, Cairo government, Egypt.

- Time: 2019 the study continued for 9 months and then all patients continued their orthodontic treatment in clinic of Orthodontic department, Faculty of Dentistry, Cairo University.

Intervention

1. Group I (Segmented tray group)

a. Patient Screening and Preparation:

i. Selection and examination of the patients according to inclusion and exclusion criteria was done and assigning each patient in his/her group after randomization.

ii. Patients were properly diagnosed and the problem list, treatment objectives and a systemized treatment plan was written in the diagnostic sheet form.

iii. Full records of the patients were taken:

- Full intra-oral photographs.

- Maxillary and mandibular study models.

- Panoramic and lateral cephalometric radiographs.

b. Scanning and digital bracket placement: CEREC Omnicam intraoral scanner was done to capture the initial position of the teeth and to construct a 3D working model on which the orthodontic attachments were placed and the transfer tray was digitally designed and fabricated. The stereolithographic (STL) file was then exported from the scanner software.

c. Fabrication of trays: By using 3 Shape Ortho analyzer Software was used to fabricate segmented digital bracket transfer tray (two segments; splitted at the midline) for patients of treatment group and full arch tray for comparative group. The following steps were done:

i. Model preparation:

- The selected scan was prepared for brackets placements through clicking on "prepare model set" option and the instructions were followed to trim it to imitate the stone model. It is then refined to remove any nodules or to fill any voids, and to define the gingival margins of all teeth.

- The contact points were defined and were put on the mesial and distal occlusal surfaces of all teeth from the upper right first molar to the upper left first molar.

- Now, the model is prepared and ready for attachments placements.

ii. Bracket's placements:

- The prescription of the brackets was chosen from the library was "American Orthodontics mini master Roth 0.22 with hooks 5-5 ver1" and the prescription of the tubes of first molar chosen was "American Orthodontics buccal tubes Roth 0.22 ver1.

- The position of brackets and tubes was adjusted by clicking on "bracket and teeth adjustments".

- Each attachment's position was then modified individually according to the investigator's preference and by the help of the digital calibrations calculated by the software in all dimensions.

- This is called "the master model" which will be used next in fabrication of the digital tray. This model was also exported in an STL format to be used as the reference or the pre-operative model (Figure 1).

iii. Tray Designing: By using 3 Shape Ortho Appliance Designer, the following were done:

- The prepared master model with attachments was selected for tray fabrication.

- The design of the tray was created through placing points to define the tray borders.

iv. Tray Printing:

- The tray was printed (Figure 2) in a flexible resin material (indirect bonding tray material), allowing the tray to be easily inserted and removed from the patient's mouth. 
- Dent 1 3D Printer (Mogassam, Cairo, Egypt) was used for tray printing, with XY resolution $50 \mathrm{um}$ and $Z$ layer thickness 25 um and capability of printing up to $3 \mathrm{~cm}$ per hour.

d. Bracket transfer using digital tray:

i. Fitting of metal brackets $(0.022 * 0.025$ inches Roth prescription, American Orhtodontics, Master Series ${ }^{\mathrm{TM}}$ ) into digital tray and ensure keeping them in place in their precise rooms created for them.

ii. The tray was sectioned by lancet at the midline to obtain two segments.

iii. Conventional procedures of indirect bonding are followed including etching, bonding and curing.

iv. The sectioned tray was then removed lightly from the patient's mouth.

v. The left section of the tray carrying orthodontic attachments was inserted, cured and then removed by the same technique.

vi. The orthodontic attachments were now ready for intraoral scanning.

2. Group II (Full Arch Tray Group)

a. Same steps of indirect bonding procedure were followed in this group patients as group I (Segmented Tray Group) but the tray fabricated was full arch tray instead of segmented one.

b. Same procedure of intraoral scanning was done as in group I (Segmented Tray Group).

c. Comparison between position of brackets on pre and post-operative scan was done.

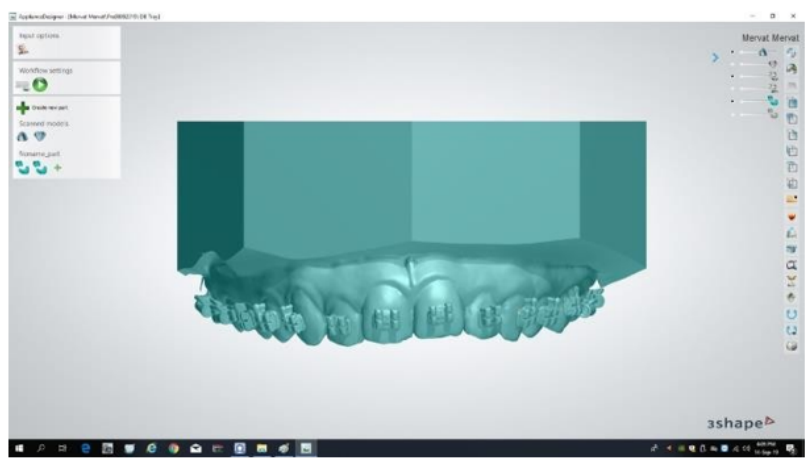

Figure 1: 3 Shape software window showing master model.

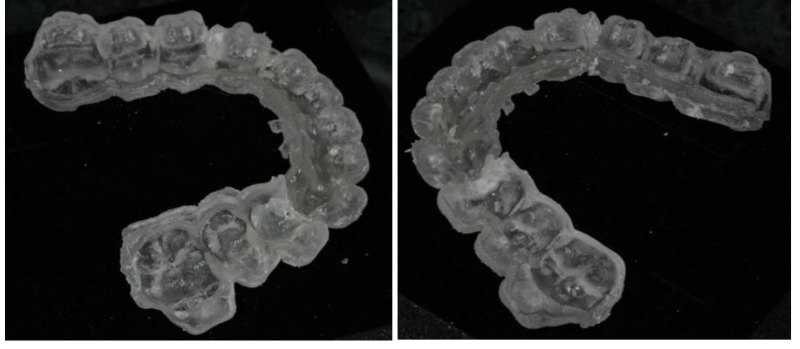

Figure 2: The 3D printed transfer tray.

Superimposition procedure:

- For every patient the pre-operative scan and post-operative scans were obtained, where the former scan was set as a reference while the latter was set as a test.

- Using 3Shape OrthoAnalyzer software, superimposition of both models was carried out.

- This superimposition showed the discrepancy between the two scans with defining areas and amount of deviation, where the areas in green color showed pre-operative scan and areas toward orange color represent any deviation of the post-operative scan away of the pre-operative one.

\section{Measurements on the software:}

- The Mesiodistal, Occluso-gingival and Bucco-lingual discrepancies were measured as the linear distance between the pre-operative and post-operative positions, X plane, Y plane and $\mathrm{Z}$ plane respectively.

- To measure the mesio-distal discrepancy, an imaginary plane is drawn perpendicular to the $\mathrm{X}$ axis. The distance from the pre-operative bracket position to this plane is measured, and the distance from the post-operative bracket position to the same plane is also measured. The Linear discrepancy is the subtract of these two readings.

- This procedure is done four times for each bracket; from each wing to the imaginary plane, and the mean distance is then calculated.

- The same steps are done to measure the occluso-gingival (and Bucco-lingual discrepancies).

\section{Results}

The statistical analysis was performed by specialized statistician using IBM SPSS Statistics Version 20 for Windows. 
The results of the trial will be presented under the following headings:

- Data normality (Table 1).

- Accuracy of transfer of orthodontic attachments in terms of mesiodistal (Table 2) and (Figure 3), occlusogingival (Table 3) and (Figure 4), buccolingual (Table 4) and (Figure 5) deviations.

\begin{tabular}{|c|c|c|c|c|}
\hline & \multirow[b]{2}{*}{$\mathbf{N}$} & \multicolumn{2}{|c|}{$P$ - value } \\
\hline & & & $\begin{array}{c}\text { Group I } \\
\text { (Segmented } \\
\text { Tray) }\end{array}$ & $\begin{array}{c}\text { Group II } \\
\text { (Full Arch } \\
\text { Tray) }\end{array}$ \\
\hline \multirow{3}{*}{$\begin{array}{l}\text { Linear } \\
\text { Measurements }\end{array}$} & $\begin{array}{c}\text { Mesio-distal } \\
\text { Deviation (X- axis) }\end{array}$ & 144 & $>0.05$ & $>0.05$ \\
\hline & $\begin{array}{c}\text { Occluso-gingival } \\
\text { Deviation (Z-axis) }\end{array}$ & 144 & $>0.05$ & $>0.05$ \\
\hline & $\begin{array}{c}\text { Bucco-lingual } \\
\text { Deviation (Y-axis) }\end{array}$ & 144 & $>0.05$ & $>0.05$ \\
\hline
\end{tabular}

Table 1: Normality exploration of each attachment on each tooth for both groups.

$\mathrm{N}$ : Attachments count.

\begin{tabular}{|l|c|c|c|}
\hline & Mesial & Distal & P-value \\
\hline Group I (Segmented Tray) & $45 \%$ & $55 \%$ & 0.631 \\
\hline Group II (Full Arch Tray) & $40 \%$ & $60 \%$ & 0.337 \\
\hline P-value & 0.808 & 0.810 & \\
\hline
\end{tabular}

Table 2: Percentages of mesial and distal deviation in group I and II.

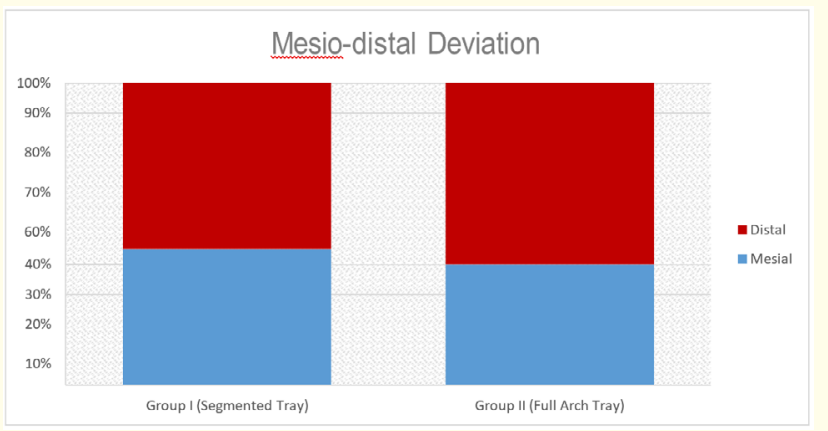

Figure 3: Bar chart percentages of mesial and distal deviation in group I and II.

\begin{tabular}{|l|c|c|c|}
\hline & Occlusal & Gingival & P-value \\
\hline Group I (Segmented Tray) & $47 \%$ & $53 \%$ & 0.337 \\
\hline Group II (Full Arch Tray) & $65 \%$ & $35 \%$ & 0.152 \\
\hline P-value & 0.384 & 0.381 & \\
\hline
\end{tabular}

Table 3: Percentages of occlusal and gingival deviation in group I and II.

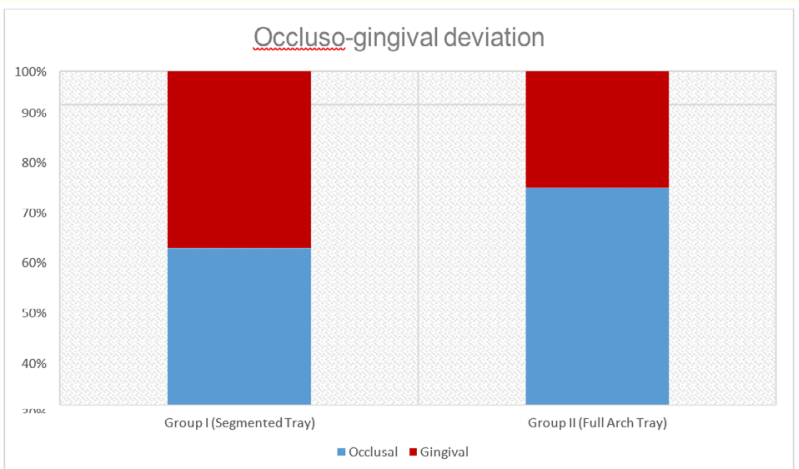

Figure 4: Bar chart percentages of occlusal and gingival deviation in group I and II.

\begin{tabular}{|l|c|c|c|}
\hline & Buccal- out & Lingual- in & P-value \\
\hline Group I (Segmented Tray) & $42 \%$ & $58 \%$ & 0.431 \\
\hline Group II (Full Arch Tray) & $47 \%$ & $53 \%$ & 0.775 \\
\hline P-value & 0.809 & 0.849 & \\
\hline
\end{tabular}

Table 4: Percentages of buccal and lingual deviations in group I and II.

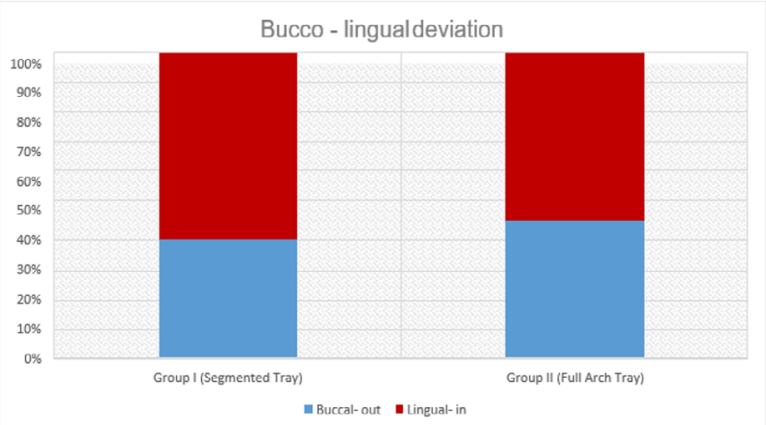

Figure 5: Bar chart percentages bucco-lingual deviation in group I and II. 


\section{Discussion}

Placement of orthodontic attachments on the patient's dentition is usually done by either a direct or an indirect bonding technique. Silverman and Cohen [7] (1972) were the first to develop indirect bonding technique to reduce clinical time and to enhance patient comfort. The indirect bonding technique has many advantages as: allowing better three-dimensional visualization of tooth positioning and, as a result, greater precision while positioning orthodontic attachments will be reached. As a result of accurate bracket placement, reduction of the need for later repositioning or complex wire bending at the finishing stage, thus shortening treatment time and improving efficiency of treatment. Furthermore, reduction of the complications accompanying orthodontic treatment will occur, such as white spot lesions and root resorption and thus patient satisfaction will be the final result.

Accuracy of bracket positioning using indirect bonding technique may be attributed to thickness of bonding material between teeth and brackets, any contamination that may occur during transfer, or any error that occurred during transfer tray fabrication. However, high accuracy of bracket positioning during transfer, was found with segmented trays, reaching 98\% regarding buccolingual and mesiodistal dimension [8].

The method used for measuring the accuracy of attachment transfer in all three planes was the method that is described by Elnigoumi [9] which was based on the reliability of 3D models in terms of linear and angular measurements. The study was carried out using digital scans and digital measurements on (Geomagic software version 12). The digital scanning had the following advantages: obtaining accurate and reproducible measurements unlike the 2D photography images that were used previously, recording minute details up to parts of microns due to the ultimate precision of intraoral scanners and finally, prevention of patient exposure to any kind of unnecessary radiation such as CBCT which was used earlier to evaluate the accuracy of indirect bonding.

Referring to the results of the present study, it was essential to mention the statistical findings of the different outcomes of the current study. Furthermore, it was essential to compare them to the findings of similar studies in the previous literature.

As for accuracy of attachment transfer, for each attachment linear and angular measurements were done. Any change in the po- sitioning of the attachment itself is recorded as deviation in the attachment position (linear and/or angular). For example, a value of $0.2 \mathrm{~mm}$ or 0.2 degree in a certain plane would reflect that the tube was bonded $0.2 \mathrm{~mm}$ or 0.2 degree away from its original position on the working model. the readings were compared relative to the accepted range of +/- $0.5 \mathrm{~mm}$ which was reported by Grunheid., et al. [10] regarding linear measurement deviation,

As for the linear measurements, mesiodistal, occlusogingival and buccolingual deviations were within $0.5 \mathrm{~mm}$ limit defined by Grunheid., et al [10]. Regarding the mesio- distal directional deviation in the present study, no statistically significant difference was found between segmented and the full arch tray techniques 0.23 $\mathrm{mm}$ and $0.29 \mathrm{~mm}$ ) respectively. Furthermore, the present study results showed an agreement with the findings of Grunheid., et al. [10] with no directional bias either towards the mesial or distal directions for both studies regarding segmented tray. Regarding the occluso-gingival directional deviation in the present study, there was no statistically significant difference between segmented and the full arch tray techniques $(0.48 \mathrm{~mm}$ and $0.58 \mathrm{~mm})$ respectively. However, regarding segmented tray, there was no directional bias either towards the gingival or occlusal directions in the present study, which did not show agreement with the results of Grunheid., et al [10]. The latter have found more percentage toward the gingival direction $(60.29 \%)$ than to the occlusal direction $(39.71 \%)$. This might be due to the stretched indirect transfer tray during the clinical bonding procedure by the operator's fingers pressing the tray gingivally. Regarding the bucco-lingual directional deviation in the present study, there was no statistically significant difference between segmented and the full arch tray techniques $0.26 \mathrm{~mm}$ and $0.30 \mathrm{~mm}$ ) respectively. However, regarding segmented tray, there was no directional bias either towards the buccal or lingual directions in the present study, which was different from that results of Grunheid., et al [10]. The results were the directional bias was towards the buccal direction (79.41\%). The reason behind the buccal directional bias might be due to the adhesive being applied to the orthodontic attachments during the clinical bonding procedure.

The findings of this study showed that the two indirect bonding techniques are accurate with the segmented tray showing significant reduction in bond failure rate. 


\section{Conclusion}

Based on the results of the clinical and statistical analyses, the following conclusions could be reached. Linear attachment deviations were within the clinically acceptable range of deviation $(+/-0.5$ $\mathrm{mm}$ ) in all three planes for both techniques. Linear directional deviation in the mesio-distal plane, occluso-gingival and bucco-lingual showed no differences between both techniques. Regarding the percentage of angular directional deviation, torqueing and rotational deviations both techniques are comparable.

\section{Bibliography}

1. Sondhi A. "Efficient and effective indirect bonding". American Journal of Orthodontics and Dentofacial Orthopedics 115 (1999): 352359.

2. Gru T., et al. "Transfer accuracy of vinyl polysiloxane trays for indirect bonding (1972).

3. Menini A., et al. "A 15-month evaluation of bond failures of orthodontic brackets bonded with direct versus indirect bonding technique: a clinical trial (2014): 1-6.

4. Cozzani M., et al. "Etching masks for precise indirect bonding". Journal of Clinical Orthodontics 44 (2010): 326-330.

5. Silverman E., et al. "A universal direct bonding system for both metal and plastic brackets". American Journal of Orthodontics and Dentofacial Orthopedics 62.3 (1972): 236-244.

6. Grünheid T., et al. "Transfer accuracy of vinyl polysiloxane trays for indirect bonding". The Angle Orthodontist 86.3 (2016): 468474.

7. El Nigoumi A. "Assessing the Accuracy of Indirect Bonding with 3D Scanning Technology 37.06 (2016): 613-619.

8. Grünheid T., et al. "Accuracy, reproducibility, and time efficiency of dental measurements using different technologies". American Journal of Orthodontics and Dentofacial Orthopedics 145.2 (2014): 157-164.

9. Schmid J., et al. "Transfer accuracy of two indirect bonding techniques-an in vitro study with 3D scanned models". The European Journal of Orthodontics 40.5 (2018): 549-555.
10. Rossini G., et al. "Diagnostic accuracy and measurement sensitivity of digital models for orthodontic purposes: A systematic review". American Journal of Orthodontics and Dentofacial Orthopedics 149.2 (2016): 161-170.

\section{Assets from publication with us}

- Prompt Acknowledgement after receiving the article

- Thorough Double blinded peer review

- Rapid Publication

- Issue of Publication Certificate

- High visibility of your Published work

Website: www.actascientific.com/

Submit Article: www.actascientific.com/submission.php

Email us: editor@actascientific.com

Contact us: +919182824667 